\title{
Middle and late latency ERP components discriminate between adults, typical children, and children with sensory processing disorders
}

\author{
Patricia L. Davies ${ }^{1}$, Wen-Pin Chang ${ }^{2}$ and William J. Gavin ${ }^{1}$ \\ ' Department of Occupational Therapy, Colorado State University, Fort Collins, CO, USA \\ 2 Krannert School of Physical Therapy, University of Indianapolis, Indianapolis, IN, USA
}

Edited by:

Sidney A. Simon, Duke University, USA

Reviewed by:

Curtis Ponton, Compumedics

Neuroscan, USA

Sidney A. Simon, Duke University, USA

*Correspondence:

Patricia L. Davies, Department of

OccupationalTherapy, 219

Occupational Therapy, Colorado State

University, Fort Collins, CO 80523,

USA.

e-mail:pdavies@lamar.colostate.edu
This study examined whether combinations of middle latency sensory evoked potential components and late components, possibly indicative of cognitive processing, can discriminate between three sample groups; 18 adults (20-55 years), 25 typical children (5-10 years) and 28 children with sensory processing disorders (SPD) (5-12 years). Electroencephalography (EEG) recordings were made while participants heard random presentations of two auditory stimuli (1 and $3 \mathrm{kHz}$ ) each at two intensities ( 50 and $70 \mathrm{~dB}$ ). Amplitude and latency measurements were obtained for the N1, P2, N2, and P3 components from the averaged event-related potential (ERP) for each of the four auditory stimuli. Discriminant analyses revealed two functions, one which described the relationship of the components on SPD deficit continuum and one which described the relationship of these components on a developmental continuum. Together, these two functions correctly classified $90.5 \%$ of the participants as to their group membership. These results are discussed in relation to neurodevelopmental theories.

Keywords: sensory registration, event-related potentials, brain maturation, auditory processing, discriminant analysis

\section{INTRODUCTION}

Electroencephalography (EEG) and event-related potentials (ERPs) provide an important bridge in studying the relationship between behavioral performance and brain structure and function (Polich, 1993). Electroencephalography records the electrical activity of the brain via electrodes placed on the scalp and provides continuous measures of brain processing in real time. Averaged ERPs can be obtained when multiple presentations of an event such as a defined auditory stimulus occur during the EEG recordings and the EEG segments surrounding the event are averaged together. Thus, ERPs represent an intricate pattern of brain processing in response to events that can range from the presentation of simple sensory stimuli to complex events which may engage decision making or reasoning. Event-related potentials reveal that when the human brain processes a simple sensory stimulus in isolation of decision making, i.e., a passive experience, much of the brain's response to the stimulus occurs in the early (e.g., $0-20 \mathrm{~ms}$ ) to middle latency (20-100 ms) periods following the stimulus, with little activity occurring after $250 \mathrm{~ms}$ following the stimulus presentation (for an example, see the classic study of Ponton et al., 2000). When the human brain processes a more complex event, such as in a decision making task, ERPs reveal that brain processing occurs for longer periods following the event. Often in these paradigms the brain processing continues past $250 \mathrm{~ms}$ following the stimulus with the observed activity referred to as late latency components. One purpose of this study was to determine if the examination of both the middle and late ERP components in auditory ERPs would better characterize differences between adults, typically developing children and children with sensory processing disorders (SPD) than just examining middle latency components alone.

\section{ERP COMPONENTS}

Event-related potentials are characterized by an intricate series of components following the event or stimulus presentation. The components are thought to be generated by one or more generator sources or dipoles which are presumed to be located in cortical tissue that operate together and have specialized functions (Segalowitz and Davies, 2004). Components are believed to be associated with particular sensory or cognitive functions (Banaschewski and Brandeis, 2007) and are described by their topography, polarity, amplitude, and latency. The principal middle to late latency components of the ERP are a positive-negative-positive-negative complex typically labeled $\mathrm{P} 1-\mathrm{N} 1-\mathrm{P} 2-\mathrm{N} 2$ and begin around $40-50 \mathrm{~ms}$ and continue for another 150-250 ms (Polich, 1993; Ponton et al., 2000). A number of studies have found that the $\mathrm{N} 1$ and $\mathrm{P} 2$ have been found to be sensitive to intensity and frequency of auditory stimuli. Specifically, N1 and P2 amplitude increase with increasing intensity of auditory stimuli (e.g., Picton et al., 1974; Adler and Adler, 1989, 1991). In addition, N1 and P2 amplitudes are larger with lower frequency stimuli compared to higher frequency stimuli (Picton et al., 1974). Generally, ERP paradigms that are passive and do not require psychological action (i.e., evaluative or motor responses) often do not show deflections following the P2 or N2. However, in some situations, especially in an ERP elicited by a cognitive paradigm the $\mathrm{N} 2$ is followed by a pronounced positive peak, labeled P3 (or sometimes labeled P300, P3a, or P3b) and typically peaks around $250-450 \mathrm{~ms}$, but the latency varies with the time required to process the event (Polich, 1993).

While the early and middle latency sensory evoked potentials are often passive and involve no response or active cognitive processing by the participant, the later ERP components involve 
cognitive processing such as evaluating a target or novel stimulus (Polich, 1993). Recent studies in children with disabilities have shown that it may be important to consider both middle and late latency components whether the paradigm is a sensory paradigm or a cognitive paradigm (Lopez et al., 2006; Davies and Gavin, 2007). Thus, considering both middle and late components in developmental ERP data may be advantageous, especially as the components and sources of the ERPs are complex (Ponton et al., 2000).

\section{MATURATION OF ERPs}

Maturation of auditory ERP components extends up to 16 years of age (e.g., Bruneau et al., 1997; Bruneau et al., 1999; e.g., Ponton et al., 2002). Auditory ERPs in young children are dominated by $\mathrm{P} 1$ and N2 components, opposed to the mature P1-N1-P2-N2 component morphology in adults (Čeponienè et al., 2002). In children the N2 is the most predominant negative peak of auditory evoked potentials, whereas in adults the N1 component dominates (Čeponienè et al., 2002). The presence of the N1 component in children is influenced by the timing between the presentation of the auditory stimuli (inter-stimulus interval, ISI). Specifically, the N1 often is not present in ERPs of young children when the paradigm's ISIs are shorter than $1 \mathrm{~s}$, but the $\mathrm{N} 1$ component is observed in children at least 9 years of age when ISIs are longer than $1 \mathrm{~s}$ (Paetau et al., 1995; Bruneau and Gomot, 1998; Čeponiené et al., 2002).

In addition to these components, evidence exists for an extended developmental time course of the $\mathrm{P} 3$, a late latency component (Segalowitz and Davies, 2004). Using a decision making task, the novelty odd-ball paradigm, Segalowitz and Davies (2004) found that younger children produce large $\mathrm{P} 3 \mathrm{~s}$ to the target stimuli with a posterior maximum, but very unclear and inconsistent results for the P3 were observed to novel stimuli while adults showed the pattern of a frontal maximum in response to novel stimuli. Segalowitz and Davies (2004) also indicated that children start to show a more standard adult pattern of the P3 around age 13. Other investigators have demonstrated that the P3 is related to diverse functions; including memory updating, active stimulus discrimination, attention allocation, and response preparation (see Key et al., 2005 for review). These functional abilities have a prolonged time course and parallel the developmental changes seen in P3 component's latency and amplitude.

\section{PURPOSE OF THIS STUDY}

The major purpose of this present study was to examine if auditory ERP components are able to differentiate between adults, typically developing children and children with SPD. Children with neurodevelopmental disorders such as autism and attention deficit hyperactive disorder (ADHD) often display difficulties in processing sensory information (Rogers and Ozonoff, 2005; Liss et al., 2006; Yochman et al., 2006). Behavioral assessments have shown that children with SPD respond differently to sensory input in everyday activities. However, the underlying neural mechanisms for these differences in behaviors are poorly understood. The association between brain function and the behaviors of children with SPD has received only limited examination to date (Davies and Gavin, 2007; Davies et al., 2009). Uncovering neural mechanisms may assist in a better understanding the nature of the disorder and may assist in guiding appropriate treatment for children with SPD. Thus, to examine whether the relationship between ERP components is sensitive to SPD we contrasted a sample of young adults and a sample of typically developing children to a sample of children who were receiving therapy for behaviors perceived as resulting from difficulties in processing sensory stimuli. Because the processing of simple auditory stimuli can be depicted as several components in an ERP, each representing a unique aspect of detecting and interpreting sensory input, comparisons of different ERP components and their relationship will be examined in this study.

Our first research question is "Do healthy adults and children with and without SPD display differences amplitudes of the ERP components in response to increased stimulus intensity or frequency?" To answer this question, we compared the mean amplitudes of the N1, P2, N2, and P3 components of the three groups using the traditional univariate approach. To explore the inter-relationship of the components we asked a second question, "Can a combination of ERP components within an individual's brain response to a stimulus be used to successfully classify an individual's membership in one of the three groups?" To address this question we used a multivariate approach, the discriminant function analysis, to classify individuals into one of the three groups based solely on the organization of ERP components. Thus, the multiple components within the ERP were used to classify the individuals into one of three groups taking advantage of the pattern of processing that differentiates the groups.

\section{MATERIALS AND METHODS PARTICIPANT}

This study consisted of three groups of participants. An adult control group comprising of 18 volunteers with equal numbers of males and females between 20 and 55 years of age $(M=33.28$; $\mathrm{SD}=11.35)$ were recruited from the local community and the university. The second group comprising of 25 typically developing children (typical; 13 male and 12 female) between 5 and 10 years of age $(M=8.33$; $\mathrm{SD}=1.88)$ were recruited from the local community through schools, youth organizations or from families who participated in past research projects in this lab. The third group consisted of 28 children with SPD ( 22 male and 6 female) between 5 and 12 years of age $(M=7.70 ; \mathrm{SD}=1.80)$ who were referred to the study by local medical practitioners. There was no significant age difference between typical children and children with SPD $(t[51]=1.25, p=0.22)$. The large number of males in this group compared to females is consistent with SPD being more prominent in males (Ahn et al., 2004).

To validate differences in sensory processing capabilities between the two child groups we used the Sensory Profile (Dunn, 1999). The Sensory Profile is a caregiver questionnaire that measures how the child processes sensory information in everyday activities. A multivariate analysis of variance on the scores on a shortened version of the Sensory Profile demonstrated that typical children had significant higher scores on five of the seven subscales and the total score of the Short Sensory Profile (Wilks' lamda $=0.43, F[7,45]=8.43, p<0.0005)$. Because a lower score indicates more sensory processing difficulties, this 
result provides face validity that the group of children referred by the medical community displayed deficits in processing sensory information.

A parent of all child participants reported no hearing deficits in their child and all adult participants reported no hearing deficits. The adult participants, based on the self-report, and typical children, based on the parent report, were free of neurological disorders, psychiatric disorders, and family histories of psychiatric disorders. For children with SPD, in addition to the primary diagnosis by a medical specialist for SPD, the parent report indicated that five children also had ADHD, two children had learning disorders (LD), five children had delayed speech, seven children had combined ADHD and LD and one child had combined LD and delayed speech. None of the children with SPD had been diagnosed as having schizophrenia, bipolar disorder, or autism or had a family history of these disorders (for a more detailed report of participant characteristics see Davies et al., 2009). The medical specialists referring the children with SPD to this study used a variety of standardized tests and clinical observations to classify the children as having SPD. Because the children were referred to our study by multiple clinics and each clinic used different assessment tools, a definitive report on the classification test results is not possible. Only six of the children with SPD were on medications; four for ADHD (2 Adderall XL, 1 Stratera, 1 specific medication not listed), one for ADHD and obsessive-compulsive disorder (Stratera and Zoloft), and one for depression ( $2.5 \mathrm{mg}$ of Abilify). We examined the data to determine if the children taking medications should be included in the study by conducting $t$-tests for the 17 variables included in the discriminant analysis. We compared the children with SPD on medications compared to the children with SPD not on medications. Only two of the resulting $t$-tests were notable; for the change amplitude for $\mathrm{P} 2$ for the $1 \mathrm{~K}$ tone at $\mathrm{Pz}\left(\mathrm{Pz} \_\mathrm{P} 2 \_\mathrm{iK} \_\right.$IntenDelta; $t[24]=2.88, p=0.008)$ and change latency for $\mathrm{P} 3$ for the $3 \mathrm{~K}$ tone at Fz (Fz_P3_3K_LatDelta; $t[24]=2.35, p=0.027)$, but when using a bonferroni correction for the number of tests these are not significant. In addition, there were no significant differences on any of the subscales or total score on the sensory profile for children with SPD taking medications as compared to those not on medications. Based on these analyses it was decided to keep the children with medications in the study.

\section{PROCEDURE}

All procedures used in this study were approved by the human research committee of the local university. Informed consent was obtained from the adult participants. For the child participants, parent permission and child assent were obtained. The participants were tested in a relaxed sitting position. Prior to EEG recording, they were provided a short training period on how to reduce artifacts that can be produced by eye blink and muscle activity. Electroencephalography recording were made during three activities, a hearing threshold screening, a modified sensory gating paradigm, and a sensory registration paradigm. The presentation of the latter two activities were counter-balanced across participants. Only the data from the sensory registration paradigm will be presented here. During the sensory registration paradigm the participants watched a silent movie and were not required to respond to the auditory stimuli.

\section{ELECTROPHYSIOLOGICAL PARADIGM AND RECORDING METHODS}

The auditory sensory registration ERP paradigm used was one adapted from Lincoln et al. (1995). Four auditory stimuli were randomly presented using the E-Prime software (Psychological Software Tools, Pittsburgh, PA, USA) to each participant binaurally through the ER-3A inserted earphones (Etymotic Research) equally distributed in four blocks of 100 stimulus presentations with an ISI of $2 \mathrm{~s}$. Each block lasted $3.4 \mathrm{~min}$ and 30-s breaks were allowed between blocks for participants to rest. The four auditory stimuli consisted of two tones of 1 and $3 \mathrm{kHz}$ presented at $50 \mathrm{~dB}$ SPL or $70 \mathrm{~dB}$ SPL for $1 \mathrm{kHz}$ tone, and at $53 \mathrm{~dB}$ SPL or $73 \mathrm{~dB}$ SPL for the $3 \mathrm{kHz}$. All stimuli had a 50-ms duration with $10-\mathrm{ms}$ rise/ fall times.

Electroencephalography activity was recorded using a BioSemi ActiveTwo EEG system (BioSemi Inc., Amsterdam, Netherlands) with 32 pin-type Ag-AgCI sintered Active-electrodes inserted into a lycra head cap with locations based upon the American Electroencephalographic Society nomenclature guidelines (1994). Electroencephalography was recorded with the Common Mode Sense (CMS) active electrode and Driven Right Leg (DRL) passive electrode as the reference and ground respectively (http://www. biosemi.com/faq/cms\&drl.htm). Electrooculograms (EOG) were recorded from individual electrodes placed on the left and right outer canthus for horizontal movements and on the left supraorbital and infraorbital region for vertical movements. Four more individual electrodes were placed on the left and right ear lobes and left and right mastoids. Electroencephalography signals were sampled at an analog-to-digit rate of $1024 \mathrm{~Hz}$ with a bandwidth of $268 \mathrm{~Hz}$.

\section{ERP WAVEFORM AND COMPONENT ANALYSIS}

All EEG/ERP analyses were conducted offline using the BrainVision Analyzer software (Brain Products GmbH, München, Germany). The left and right earlobe recordings were averaged and used as the offline reference. The four individual EOG channels were converted to a vertical and a horizontal bipolar EOG. The EEG recordings were filtered with a band pass of $0.23-30 \mathrm{~Hz}$ ( $12 \mathrm{~dB} /$ octave). The EEG was segmented about each auditory stimulus with a duration of 100 -ms pre-stimulus onset to 800 -ms post-stimulus onset. Segments with deviations greater than $\pm 100 \mu \mathrm{V}$ on any of the EEG channels or the bipolar EOG channels were eliminated. Then nonrejected segments for each auditory stimulus were baseline corrected and averaged to create ERP waveforms for each participant from which the ERP components were measured. The average number of non-rejected trials of each of the four auditory stimuli for adults were 91.93 ( $\mathrm{SD}=8.46$ ); for the typical children were $60.65(\mathrm{SD}=22.00)$, and for the children with SPD were 41.45 $(\mathrm{SD}=17.89)$. Two typical children and one child with SPD were excluded from the ERP component analysis due to an insufficient number of non-rejected segments.

Measures of peak-to-peak amplitude and latency for P1, N1 and P2 were obtained in a manner outlined by Lincoln et al. (1995) but with slight adjustments to time windows to accommodate adult ERPs. The N1 was identified between 70 and $170 \mathrm{~ms}$ and the P1 was scored between 20 and $80 \mathrm{~ms}$. The peak-to-peak amplitude of the $\mathrm{N} 1$ component was defined as the difference in $\mu \mathrm{V}$ between the $\mathrm{N} 1$ peak amplitude and the $\mathrm{P} 1$ peak amplitude. The $\mathrm{P} 2$ component 
was identified as the most positive peak between 130 and $270 \mathrm{~ms}$ after the stimulus onset and peak-to-peak amplitude was defined as the difference in $\mu \mathrm{V}$ between the $\mathrm{N} 1$ peak and the $\mathrm{P} 2$ peak. The window for determining the N2 and P3 peaks was based on the visual inspection of the grand average waveforms for adults and children. The N2 component was identified as the most negative peak between 200 and $375 \mathrm{~ms}$ after the stimulus onset and the peak-to-peak amplitude was defined as the difference in amplitude between the P2 peak and the N2 peak. The P3 component was identified as the most positive peak between 250 and $450 \mathrm{~ms}$ after the stimulus onset. The peak-to-peak amplitude of the P3 component was defined as the amplitude difference between the N2 peak and the P3 peak.

The amplitude and latency measurements of all components were measured at $\mathrm{Fz}, \mathrm{Cz}$, and $\mathrm{Pz}$ electrode sites for each averaged ERP waveform using a computer program, ERPScore (Segalowitz, 1999), which allowed for both the automatic scoring of peak amplitude and latency within a set window and visual inspection of the average waveform. Several components could not be identified for three typical children and one child with SPD, thus those components were not scored and considered missing data in the statistical analyses.

\section{STATISTICAL ANALYSIS}

In keeping with traditional approaches to evaluate the differences between adult participants, typical children, and children with SPD, the peak-to-peak amplitudes of the N1, P2, N2, and P3 components were each evaluated using a $3 \times 3 \times 2 \times 2$ analysis of covariance (ANCOVA). The between factor was Group (three levels: adult, typical children and SPD children). The three within factors were Site (three levels: $\mathrm{Fz}, \mathrm{Cz}$ and $\mathrm{Pz}$ ), Intensity (two levels: high vs. low) and Frequency (two levels: $1 \mathrm{kHz}$ vs. $3 \mathrm{kHz}$ ). The number of segments in the averaged ERP from which the dependent measure (i.e., the peak-to-peak amplitude) was derived served as a covariate. When necessary, Greenhouse-Geisser corrections were used to adjust for violation of the assumption of homogeneity of variances. Since there were four ANCOVA analyses, test-wise alpha level was adjusted to $p<0.0125(0.05 / 4)$ to reduce possible inflation of Type $I$ error.

Instead of relying on inferential logic to summarize the results of a series of post hoc $t$-tests which could be used examine the nature of the main effects found in each of the ANCOVAs, discriminant analyses procedures were used. Discriminant analyses allowed for the statistical determination of both the relative importance of each component in defining the groups and the nature of the relationship between each these components. The independent variables were combinations of both peak-to-peak amplitude and peak latency differences of the ERP components between loud and soft auditory stimuli at both $\mathrm{Fz}$ and $\mathrm{Pz}$ sites based upon the results of the ANCOVA analyses. The number of segments included in the averaged ERP was also included as an independent variable. The resulting classification functions were evaluated with Wilks' lambda. Additionally, the leave-one-out cross-validation method was used to evaluate the results of the percent correct classification data obtained from the discriminant analyses. The leave-one-out cross-validation procedure determined the discriminant function based on $N-1$ participants and then used the function to classify the participant left out. All statistical analyses were performed using the Statistical Package for Social Sciences (SPSS) for Windows software, 14.0 version.

\section{RESULTS}

The grand average ERP waveforms for each auditory stimulus were overlaid and shown separately for the adults, typical children, and children with SPD groups for the Fz, Cz, and Pz sites in Figure 1. The mean and standard deviation of the peak-to-peak amplitudes of the N1, P2, N2, and P3 components for each group are shown in Table 1. Visual inspection of these ERPs illustrate that the adult group displayed an organized pattern of brain activity sensitive to changes in frequency and intensity of the stimuli, while typical children demonstrated a less organization pattern as compared to the adults. Children with SPD displayed the most disorganization in their patterns of brain activity to auditory stimuli differing in intensity and frequency. In general, when compared to adults, children demonstrated smaller peak-to-peak amplitudes in both $\mathrm{N} 1$ and P2 components and in some cases showed less distinction between loud and soft stimuli. Conversely, children demonstrated larger peak-to-peak amplitudes in both N2 and P3 components and in some cases demonstrated more distinction between loud and soft stimuli when compared to adults. This pattern was seen in typical children and was even more marked in children with SPD.

\section{ANCOVA RESULTS EVALUATING PEAK-TO-PEAK AMPLITUDES OF EACH ERP COMPONENT}

The ANCOVA evaluating the N1 component revealed that none of the main effects were statistically significant. However, several interaction effects, Group $\times$ Frequency $(F[2,60]=4.07, p=0.022)$ and Group $\times$ Intensity $\times$ Frequency $(F[2,60]=3.27, p=0.045)$, approached significance but failed to meet the adjusted test-wise alpha criterion.

The ANCOVA evaluating the P2 component revealed statistically significant main effects for Group $(F[2,60]=6.03$, $p=0.004)$ and the Group $\times$ Site interaction $(F[4,120]=7.43$, $p<0.0005)$. The Site $\times$ Intensity interaction approached significance $(F[2,120]=4.09, p=0.019)$. Thus, the three groups differed in their peak-to-peak amplitudes and topographical distribution of the P2 component. Additionally, the stimulus intensity may differentially impact the peak-to-peak amplitude of the P2 component across electrode sites.

Analysis of the N2 component revealed a statistically significant main effect for Site $(F[2,118]=5.74, p=0.005)$. Again, several interactions approached significance; Group $\times$ Site $(F[4,118]=2.53$, $p=0.048)$, Site $\times$ Intensity $(F[2,118]=3.95, p=0.024)$ and Site $\times$ Frequency $(F[2,118]=3.38, p=0.042)$. These results indicate that the peak-to-peak amplitudes of the N2 component were different across electrode sites. Additionally, the stimulus intensity and frequency may differentially affect the peak-to-peak amplitude of the N2 component across electrode sites.

Analysis of the P3 component revealed that none of the main effects were statistically significant. However, the main effect for Group $(F[2,62]=3.17, p=0.049)$ and the Group $\times$ Site interaction $(F[4,124]=3.20, p=0.028)$, Group $\times$ Intensity interaction $(F[2,62]=3.93, p=0.025)$, and Site $\times$ Intensity interaction 

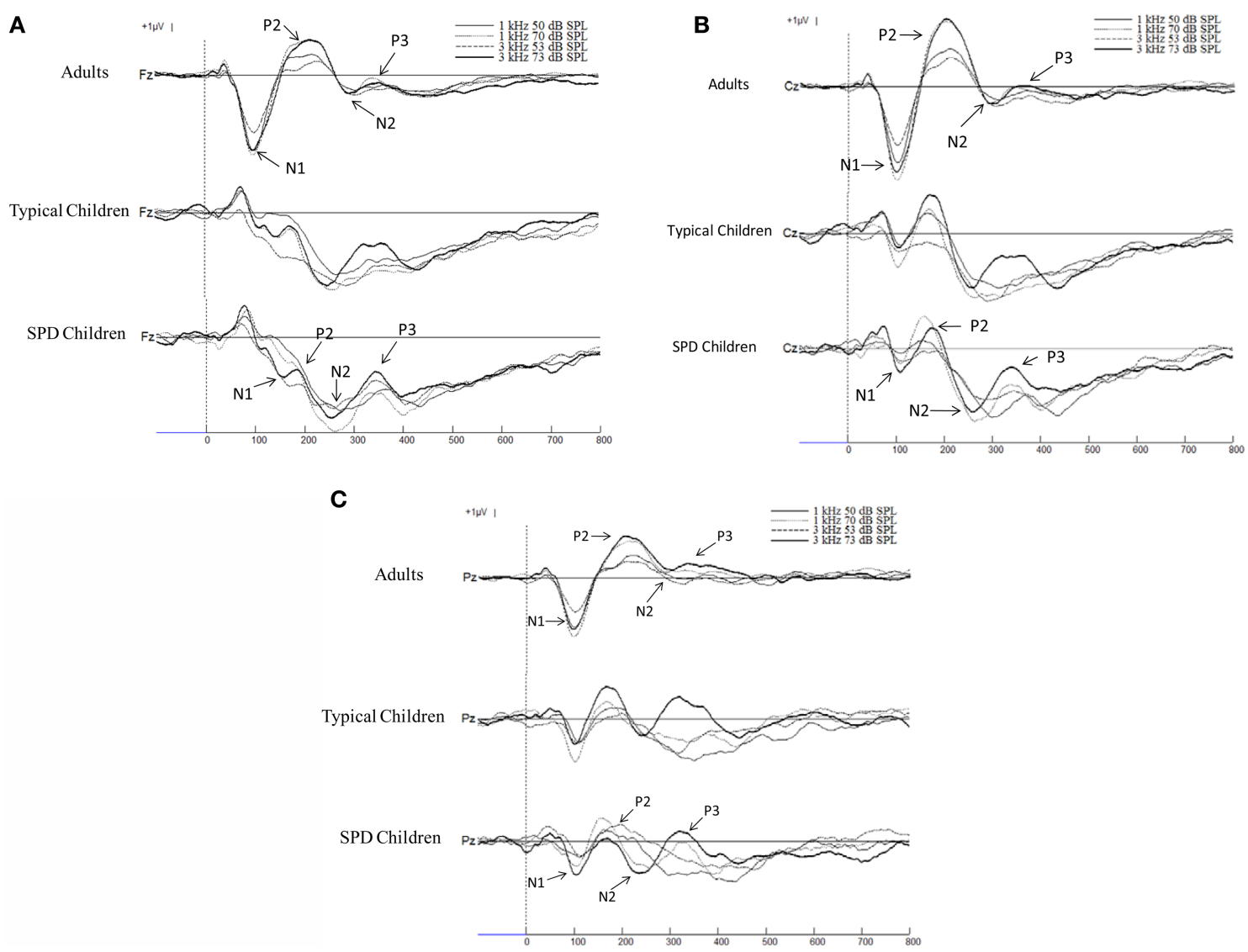

FIGURE 1 | (A) Grand averages of the ERPs at Fz with 100-ms baseline prior to the stimulus filtered with a bandpass of $0.23-30 \mathrm{~Hz}$. The thin line represents the auditory stimulus presented at $1 \mathrm{kHz} 50 \mathrm{~dB} S P L$, the dotted line represents the $1 \mathrm{kHz} 70 \mathrm{~dB}$ SPL stimulus, the dashed line represents the $3 \mathrm{kHz} 53 \mathrm{~dB}$ SPL stimulus, the thick line represents the $3 \mathrm{kHz} 73 \mathrm{~dB}$ SPL stimulus. Positive voltage is up. (B) Grand averages of the ERPs at $\mathrm{Cz}$ with $100-\mathrm{ms}$ baseline prior to the stimulus filtered with a bandpass of $0.23-30 \mathrm{~Hz}$. The thin line represents the auditory stimulus presented at $1 \mathrm{kHz} 50 \mathrm{~dB} S P L$, the dotted line represents the $1 \mathrm{kHz} 70 \mathrm{~dB}$ SPL stimulus, the dashed line represents the $3 \mathrm{kHz} 53 \mathrm{~dB}$ SPL stimulus, the thick line represents the $3 \mathrm{kHz} 73 \mathrm{~dB}$ SPL stimulus. Positive voltage is up. (C) Grand averages of the ERPs at Pz with 100-ms baseline prior to the stimulus filtered with a bandpass of $0.23-30 \mathrm{~Hz}$. The thin line represents the auditory stimulus presented at $1 \mathrm{kHz} 50 \mathrm{~dB}$ SPL, the dotted line represents the $1 \mathrm{kHz} 70 \mathrm{~dB}$ SPL stimulus, the dashed line represents the $3 \mathrm{kHz} 53 \mathrm{~dB}$ SPL stimulus, the thick line represents the $3 \mathrm{kHz} 73 \mathrm{~dB}$ SPL stimulus. Positive voltage is up.
$(F[2,124]=3.61, p=0.036)$ approached significance. These results suggest that the three groups may differ in their P3 component peak-to-peak amplitudes.

Furthermore, the peak-to-peak amplitude of the P3 component across the electrode sites of each group may be differentially affected by stimulus intensity.

\section{DISCRIMINANT ANALYSES}

To determine the relative the importance of each component in defining the groups and the nature of the relationship between each these components, two discriminant analyses were performed. To reduce the number of independent variables (i.e., input variables) and retain the information about the organization of brain responses to intensity shifts of the stimuli, the various peak-to-peak amplitudes values for P2 and P3 for the two different tones $(1$ and $3 \mathrm{kHz})$ at each of two recording sites $(\mathrm{Fz}$, $\mathrm{Pz}$ ) were collapsed by computing their difference scores between loud and soft intensities. For example, the peak-to-peak amplitude of $\mathrm{P} 2$ at Fz for the soft intensity was subtracted from the peak-to-peak amplitude of P2 at Fz for the loud intensities of $1 \mathrm{kHz}$ stimuli (I $\Delta \mathrm{P} 2$ for $1 \mathrm{kHz}$ at $\mathrm{Fz}$ ). A similar approach was used to collapse latency measures. For example, the latency of $\mathrm{P} 2$ at $\mathrm{Fz}$ for the soft intensity was subtracted from the latency of $\mathrm{P} 2$ at $\mathrm{Fz}$ for the loud intensities of $1 \mathrm{kHz}$ stimuli (L $\Delta \mathrm{P} 2$ for $1 \mathrm{kHz}$ at $\mathrm{Fz}$ ). Thus, the independent variables entered simultaneously in the discriminant analysis were the square root of the number of segments in the ERP and the 16 variables ( 8 representing amplitude response characteristics and 8 representing latency characteristics) computed in above manner (see Table 2 for complete listing).

The first discriminant analysis performed evaluated differences between the three groups; the adults, typical children and children with SPD. The results revealed that $91 \%$ of the participants could be correctly classified as their group membership by brain responses alone. The adult participants were $94 \%$ correctly classified, while $80 \%$ typical children were correctly classified and $96 \%$ children with SPD were correctly classified (see Figure 2). One adult was incorrectly classified as a typical child. Only four typical children were 
Table 1 | Mean peak-to-peak amplitude (in $\mu$ V) of the ERPs components for each auditory stimulus for each of the three groups. Standard deviations are shown in parentheses.

\begin{tabular}{|c|c|c|c|c|c|c|c|c|c|}
\hline \multirow{2}{*}{ Auditory stimulus } & \multicolumn{3}{|c|}{ Group } & & & & & & \\
\hline & \multicolumn{3}{|c|}{ Adult } & \multicolumn{3}{|c|}{ Typical children } & \multicolumn{3}{|c|}{ SPD children } \\
\hline \multicolumn{10}{|l|}{$1 \mathrm{kHz} 50 \mathrm{~dB}$ SPL } \\
\hline N1 & $9.83(3.12)$ & $10.37(3.15)$ & 7.19 (2.29) & 7.37 (3.98) & $6.70(3.90)$ & $5.79(3.25)$ & $7.29(5.05)$ & $5.39(3.43)$ & 4.65 (3.23) \\
\hline P3 & $2.19(1.10)$ & $2.74(2.09)$ & $2.72(1.36)$ & $6.59(3.94)$ & $5.89(2.56)$ & $4.97(3.02)$ & $6.94(3.70)$ & $6.47(3.96)$ & 5.18 (3.36) \\
\hline \multicolumn{10}{|l|}{$1 \mathrm{kHz} 70 \mathrm{~dB}$ SPL } \\
\hline N1 & $11.62(4.40)$ & $13.11(4.78)$ & $8.59(3.32)$ & $11.07(4.94)$ & $9.41(4.25)$ & $7.58(3.48)$ & $9.48(4.90)$ & $7.63(3.33)$ & $6.65(3.07)$ \\
\hline P2 & $15.57(6.58)$ & $20.78(7.37)$ & $13.32(4.48)$ & $6.29(4.75)$ & $11.40(8.58)$ & $11.06(7.15)$ & $5.50(4.37)$ & $10.45(8.19)$ & $9.61(6.20)$ \\
\hline N2 & $9.03(4.29)$ & $12.51(5.33)$ & $6.84(3.35)$ & $11.30(5.39)$ & $15.71(7.23)$ & $10.62(5.51)$ & $13.45(7.83)$ & $16.43(10.84)$ & $10.23(6.53)$ \\
\hline $\mathrm{P} 2$ & $9.89(4.59)$ & $11.59(4.51)$ & $7.67(2.95)$ & $3.88(2.81)$ & $6.63(4.68)$ & 8.26 (3.79) & $5.24(4.43)$ & $7.05(4.51)$ & $8.04(5.26)$ \\
\hline N2 & $6.21(3.33)$ & $7.99(4.30)$ & $4.59(2.15)$ & $7.92(5.31)$ & $11.95(5.83)$ & $9.07(5.08)$ & $9.13(5.64)$ & $11.10(5.67)$ & $8.13(5.21)$ \\
\hline P3 & $2.92(2.04)$ & $3.59(2.86)$ & $2.86(1.78)$ & $7.55(4.75)$ & $7.43(5.07)$ & $6.56(4.55)$ & $7.53(4.64)$ & $6.16(3.27)$ & $4.80(2.45)$ \\
\hline \multicolumn{10}{|l|}{$3 \mathrm{kHz} 73 \mathrm{~dB}$ SPL } \\
\hline N1 & $10.66(4.20)$ & $12.23(4.78)$ & $8.38(3.07)$ & $9.43(6.04)$ & $8.81(4.78)$ & $6.98(3.73)$ & $10.81(5.06)$ & $9.07(4.84)$ & $8.02(3.40)$ \\
\hline P2 & $14.56(6.45)$ & $19.73(8.96)$ & $12.76(4.94)$ & $5.78(5.09)$ & $11.13(8.21)$ & $10.21(5.42)$ & $5.92(4.69)$ & $10.59(6.82)$ & $8.44(5.17)$ \\
\hline $\mathrm{N} 2$ & $8.58(4.80)$ & $12.52(7.91)$ & 7.12 (3.57) & $10.44(6.83)$ & $15.49(8.59)$ & $10.33(6.19)$ & $11.11(7.46)$ & $14.60(7.80)$ & $8.77(4.72)$ \\
\hline P3 & $3.18(2.02)$ & 4.97 (3.83) & $3.95(2.18)$ & $8.17(6.04)$ & $7.73(5.82)$ & $8.60(4.87)$ & $9.19(3.73)$ & 8.98 (5.73) & $9.09(5.81)$ \\
\hline
\end{tabular}

incorrectly classified; two participants were labeled as members of the adult group and two as the SPD group). Only one child with SPD was incorrectly classified into the typical child group. Function 1 significantly separated the three groups with the centroid for the adults being on one end and the centroid for the children with SPD on the other end with centroid for the typical children in the middle between them $(\lambda=0.187, p<0.0001)$. Function 2 was not statistically significant $(\lambda=0.683, p=0.227)$, though as Figure 2 illustrates, this function separated the typical children and adults with good accuracy. However, children with SPD were dispersed along this axis across the range of values observed for adults and typical children. The cross-validation results for this first discriminant analysis using the leave-one-out procedure revealed that the overall correct classification accuracy dropped to $68 \%$ with $78 \%$ for the adults, $45 \%$ for typical children, and $80 \%$ for children with SPD.

Because the adults were almost completely separated from both child groups in the first discriminant analysis and that the adults heavily influenced the weighting in the first function which might have restricted the ability of function 2 of the analysis to clearly separate the two child groups, a second discriminant analysis focusing only on the two child groups was conducted. The independent variables were the same as described for the previous analysis. The results indicate that typical children and children with SPD were significantly separated from each other, $\lambda=0.303, p=0.001$ (see Table 2). The discriminant analysis correctly classified $97.8 \%$ of the child participants with $95 \%$ correct classification for typical children and 100\% correct classification for children with SPD. Only one of the typical children was incorrectly classified into the SPD group, the same child that was incorrectly classified in the first discriminant analysis. All of the children with SPD are correctly classified. The cross-validation results for the discriminant analysis with two child groups revealed that the correct classification accuracy rate dropped to $80 \%$ with $75 \%$ for typical children and $84 \%$ for children with SPD.

Because the slight differences in the distribution of the ages in each child group, a third discriminant analysis was performed with age included with the original independent variables to test if an alternate outcome occurs. The addition of the age of the children did not change the significance of the discrimination function (i.e., $\lambda=0.293, p=0.001$ ) nor does it substantially change the standardized discriminant function coefficients as shown in the last column of Table 2. The standardize coefficient for the age variable was -0.315 indicating age was not a major variable in the classification equation. One final discriminant analysis was performed with gender included in order to test if gender differences between the child groups alter the outcome. As with age, the addition of the gender also did not impact the significance of the discrimination function (i.e., $\lambda=0.287, p=0.001$ ) nor does it substantially change the standardized discriminant function coefficients. The standardize coefficient for the gender variable was 0.337 suggesting that gender also does not serve as a major variable in the classification equation. 


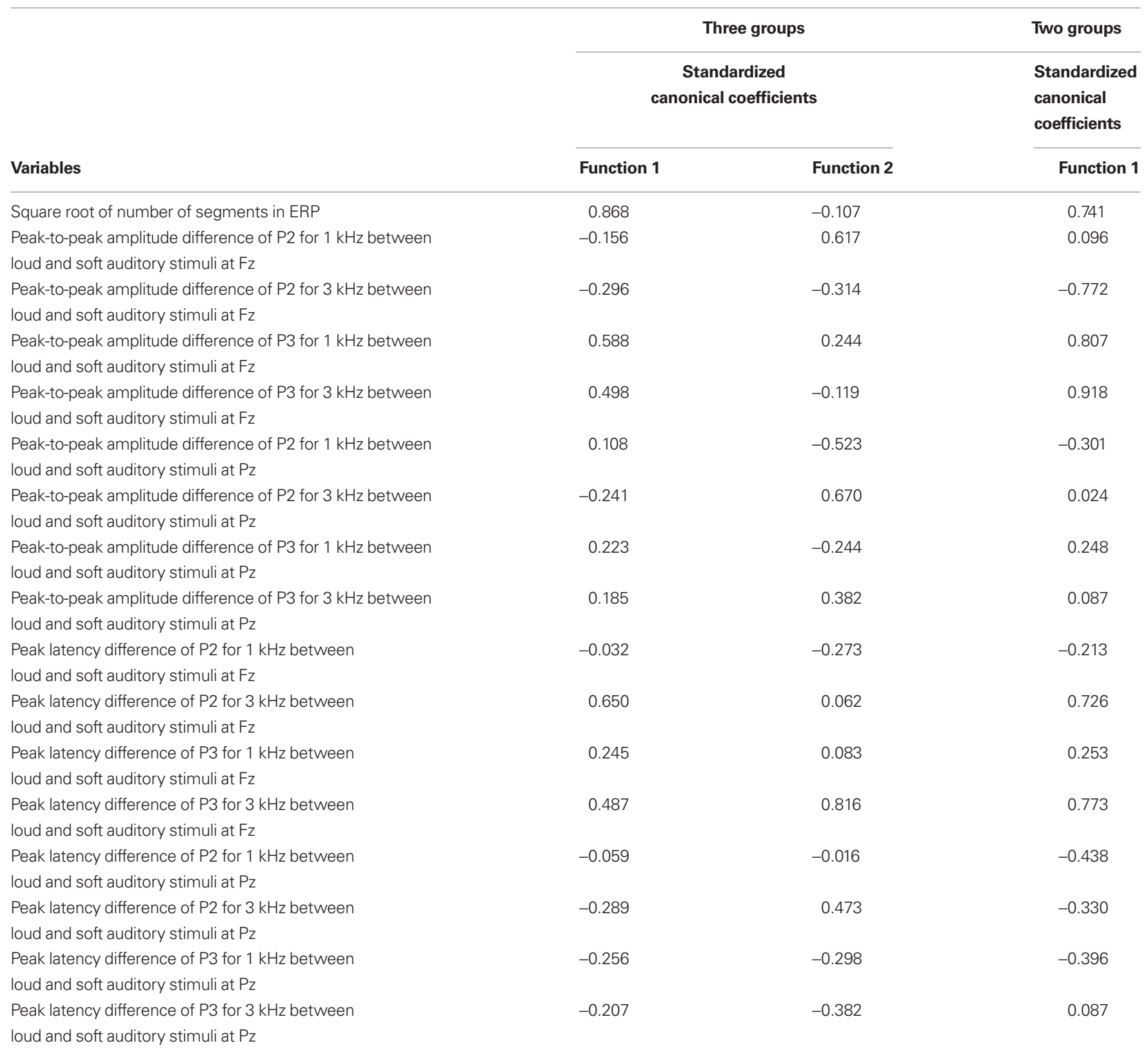

\section{DISCUSSION}

The major purpose of this present study extend the understanding of the functional role of various sensory evoked potential components and late latency cognitive components in an ERP by demonstrating that these components when considered simultaneously could differentiate adults, typical children and children with SPD. We examined the degree to which higher intensity tones ( $70 \mathrm{~dB}$ SPL) elicit a larger brain response relative to the brain response of the lower intensity tones ( $\sim 50 \mathrm{~dB}$ SPL) and the degree to which the higher intensity tones shift the peak latency relative to lower intensity tones. Sensory evoked potential components were represented by the peakto-peak measure of P2 as it was derived as the peak amplitude of N1 minus the amplitude of $\mathrm{P} 2$. The later cognitive ERP components were represented by the peak-to-peak measure of $\mathrm{P} 3$ as it was derived as the peak amplitude of $\mathrm{N} 2$ minus the amplitude of $\mathrm{P} 3$. Changes in the amplitude of these components to the intensity differences were evaluated at midline for the frontal $(\mathrm{Fz})$ and parietal $(\mathrm{Pz})$ site.

The discriminant analyses differentiated the groups of participants, adults, typical children and children with SPD with high accuracy revealing important inter-relationships between components. Specifically, the discriminant analyses revealed two functions, one which describes the relationship of the components on sensory processing deficit continuum (see Function 1 in Figure 2) and one which describes the relationship of these components on a developmental continuum (see Function 2 in Figure 2). The second function separated the adults from typical children. A primary aspect 


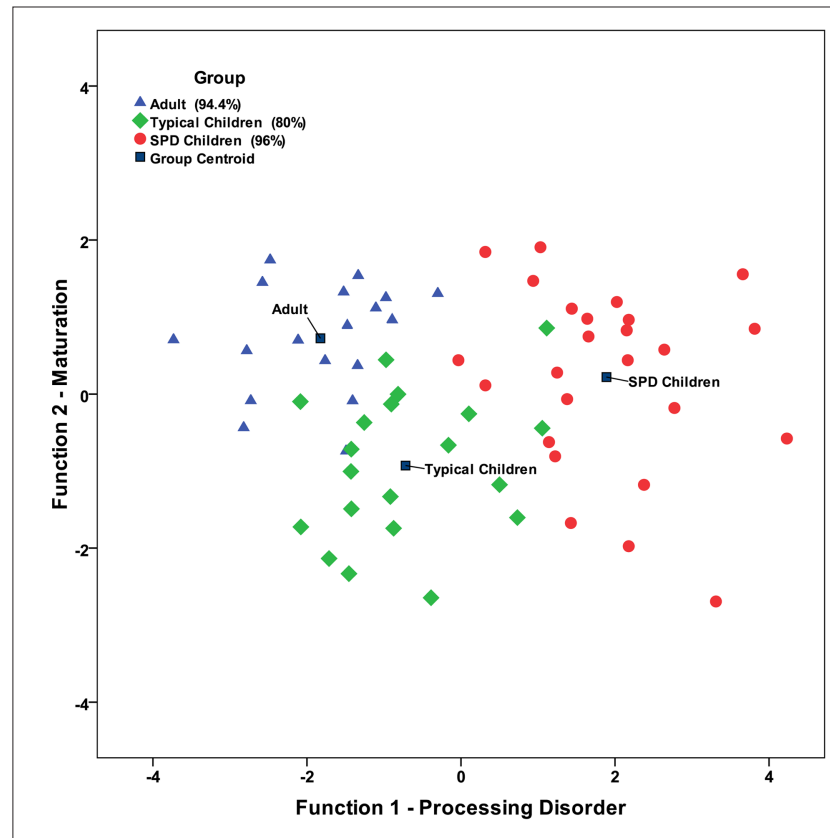

FIGURE 2 | Scatter plot for the full discriminant analysis model.

of that function involved the amplitude of components occurring around $100-150 \mathrm{~ms}$ following the presentation of stimuli (i.e. N1, $\mathrm{P} 1)$. The amplitudes of $\mathrm{N} 1$ were smaller in children compared to adults, and thus the results of this study suggest that these components increase in amplitude with maturation. This is consistent with previous research (Ponton et al., 2000; Čeponienè et al., 2002). Ponton and colleagues emphasized that the maturation of the N1 amplitude possibly relates to changes in mean synaptic density in the primary auditory cortex. Huttenlocher and Dabholkar (1997) reported that the synaptic density in the auditory cortex reaches a maximum level at about 3 months of age and then decreases approximately $60 \%$ to reach adult levels by around 12 years of age. Näätänen and Picton (1987) propose that at least six different brain processes contribute to the $\mathrm{N} 1$ component. Thus, the $\mathrm{N} 1$ component is very complex and may be influenced by several brain regions, including the frontal cortex so the primary auditory cortex may be one of several contributors to the maturational aspects of the N1.

The N1 is likely to reflect integrative and facilitative processes, sound detection, orienting, and selective attention, specifically attentional resources allocated to a relevant stimulus (Loveless, 1983; Näätänen and Picton, 1987; Näätänen, 1990; Polich, 1993; Čeponienè et al., 2002). Attending early to a stimulus is important for identifying a stimulus and determining whether or not further processing of the stimulus is required. Our findings suggest that young children have fewer resources allocated to attending to incoming stimuli, demonstrated by a smaller N1, compared to adults. This suggests that young children do not demonstrate mature sensory processing of brief auditory stimuli. More explicitly, children do not demonstrate proficiency in automatically registering external sensory stimuli, detecting stimuli and immediately determining if stimuli can be dismissed or if they need further processing. Our data suggest that children with SPD have a smaller N1 response to auditory stimuli compared to adults and their peers.
The first function in the first discriminant analysis with the three groups and the second discriminant analysis on just the two child groups demonstrated that the $\mathrm{P} 3$ was an important contributor to distinguishing between the two child groups. Distinctively, children with SPD had a larger P3 amplitude when compared to age-matched peers and adults. Altogether, these results suggest that children with SPD are not able to dismiss a sensory experience with early attention, and detection, around $100 \mathrm{~ms}$ following the event, but continue to process the information much longer and more intensely, $300 \mathrm{~ms}$ or more following the event. Thus, if sensory information is coming quickly and in multiple modalities it is easy to understand why children with SPD that actively process each sensory stimulus longer and more intensely than their peers may become overwhelmed when faced with everyday sensory experiences.

This interpretation of these data, are consistent with neurodevelopmental theories. First, the auditory brain responses are parallel with neuromaturation of auditory cortical brain region (Huttenlocher and Dabholkar, 1997; Ponton et al., 2000). Secondly, the elimination of synapses that is occurring during the development period that was examined in this study seems to be to some extent regulated by environmental experiences (Huttenlocher and Dabholkar, 1997). In agreement with this neuromaturational assumption, as children experience novel sensory experiences, they are not always able to immediately recognize sensory stimuli and so they will further process the stimuli to determine if behavioral responses are required. As children experience sensations repeatedly, they are able to quickly register the stimuli and identify whether or not further processing is needed. This may be one of the processes involved in the sculpting of synapses (i.e., synaptic elimination). We propose that in children with SPD, the maturation of the sensory systems fail and the early registration and detection of the stimuli are not developed even with repeated experiences of everyday sensory experiences. As a consequence, children with SPD are continually encountering sensory experiences as if they are always novel situations and require labored processing with each occurrence. This is consistent with Hilyard et al. (1978) proposition that the $\mathrm{N} 1$ represents the interaction between the individual and the environment, with the environment dictating what stimuli exist and the individual considering the stimuli based on personal experiences and memory. In children with sensory processing difficulties, they may lack the personal feature of the N1.

In conclusion, the results of this study support that the children that were referred to medical professionals for services due to behavioral manifestations of sensory processing difficulties demonstrate significantly different brain activity associated with simple auditory stimuli when compared to their age-matched peers and adults. By using a multivariate approach to analyses in this study we were able to correctly classify children with SPD from age-matched peers with better than $97 \%$ accuracy by using neurophysiological measures alone. These findings suggest that children with SPD exhibit less early sensory detection of stimuli which is followed by more extended and intense processing than shown by their agematched peers. The use of multivariate approaches to describe the inter-relationship between brain processing components may provide better means of identifying children with SPD and developing necessary intervention strategies. 


\section{ACKNOWLEDGMENTS}

This study was funded in part by grants from the Wallace Research Foundation and NICHD (R03HD049532) to Patricia L. Davies and William J. Gavin and by Helen F. McHugh Graduate Fellowship to

\section{REFERENCES}

Adler, G., and Adler, J. (1989). Influence of stimulus intensity on AEP components in the 80 - to 200 -millisecond latency range. Audiology 28, 316-324.

Adler, G., and Adler, J. (1991). Auditory stimulus processing at different stimulus intensities as reflected by auditory evoked potentials. Biol. Psychiatry 29, 347-356.

Ahn, R. R., Miller, L. J., Milberger, S., and McIntosh, D. N. (2004). Prevalence of parents' perceptions of sensory processing disorders among kindergarten children. Am. J. Occup. Ther. $58,287-293$.

American Electroencephalographic Society. (1994). Guidelines for standard electrode position nomenclature. J. Clin. Neurophysiol. 11, 111-113.

Banaschewski, T., and Brandeis, D. (2007). Annotation: what electrical brain activity tells us about brain function that other techniques cannot tell us - a child psychiatric perspective. J. Child Psychol. Psychiatry 48, 415-435.

Bruneau, N., and Gomot, M. (1998). "Auditory evoked potentials (N1 wave) as indices of cortical development through childhood," in Neuroimaging in Child Neuropsychiatric Disorders, ed. B. G. J. L. Beaulieu (New York: Springer), 113-124.

Bruneau, N., Roux, S., Adrien, J. L., and Barthelemy, C. (1999). Auditory associative cortex dysfunction in children with autism: evidence from late auditory evoked potentials (N1 wave- $\mathrm{T}$ complex). Clin. Neurophysiol. 110, 1927-1934.

Bruneau, N., Roux,S., Guerin,P., Barthelemy, C., and Lelord, G. (1997). Temporal prominence of auditory evoked potentials (N1 wave) in 4-8-year-old children. Psychophysiology 34, 32-38.

Ceponienè, R., Rinne, T., and Näätänen, R. (2002). Maturation of cortical sound processing as indexed by event-related potentials. Clin. Neurophysiol. 113 , 870-882.

Davies, P. L., Chang, W.-P., and Gavin, W. J. (2009). Maturation of sensory gating performance in children with and without sensory processing disorders. Int. J. Psychophysiol. 72, 187-197. doi: 10.1016/j.ijpsycho.2008.12.007.

Davies, P. L., and Gavin, W. J. (2007). Validating the diagnosis of sensory processing disorders using EEG technology. Am. J. Occup. Ther. 61, 176-189.

Dunn, W. (1999). The Sensory Profile: Examiner's Manual. San Antonio, TX: Psychological Corporation.

Hillyard, S. A., Picton, T. W., and Regan, D. (1978). "Sensation, perception and attention: analysis using ERPs," in Event-Related Brain Potentials in Man, eds E. Calloway, P. Tueting, and S. H. Koslow (New York: Academic Press), 223-321.

Huttenlocher, P. R., and Dabholkar, A. S. (1997). Regional differences in synaptogenesis in human cerebral cortex. $J$. Comp. Neurol. 387, 167-178.

Key, A. P. F., Dove, G. O., and Maguire, M. J. (2005). Linking brainwaves to the brain: an ERP primer. Dev. Neuropsychol. 27, 183-215.

Lincoln, A. J., Courchesne, E., Harms, L. and Allen, M. (1995). Sensory modulation of auditory-stimuli in children with autism and receptive developmental language disorder - eventrelated brain potential evidence. $J$. Autism Dev. Disord. 25, 521-539.

Liss, M., Saulnier, C., Fein, D., and Kinsbourne, M. (2006). Sensory and attention abnormalities in autistic spectrum disorders. Autism 10, 155-172.

Lopez, V., Lopez-Calderon, J., Ortega, R., Kreither, J., Carrasco, X., Rothhammer, P., Rothhammer, F., Rosas, R., and

Wen-Pin Chang. We appreciate the assistance of Lisa Fyffe, MS, OTR, who was very helpful in recruiting children with SPD. Finally, we wish to thank all of the children and their families whose participation made this study possible.

Aboitiz, F. (2006). Attention-deficit hyperactivity disorder involves differential cortical processing in a visual spatial attention paradigm. Clin. Neurophysiol. 117, 2540-2548.

Loveless, N. (1983). “The orienting response and evoked potentials in man," in Orienting and Habituation: Perspectives in Human Research, ed. D. Siddle (Chichester; New York: Wiley), 71-108.

Näätänen, R. (1990). The role of attention in auditory information processing as revealed by event-related potentials and other brain measures of cognitive function. Behav. Brain Sci. 13, 201-288.

Näätänen, R., and Picton, T. (1987). The N1 wave of the human electric and magnetic response to sound: a review and an analysis of the component structure. Psychophysiology 24, 375-425.

Paetau, R., Ahonen, A., Salonen, O. and Sams, M. (1995). Auditory evoked magnetic fields to tones and pseudowords in healthy children and adults. J. Clin. Neurophysiol. 12, 177-185.

Picton, T. W., Hillyard, S. A., Krausz, H. I. and Galambos, R. (1974). Human auditory evoked potentials: I. Evaluation of components. Electroencephalogr. Clin. Neurophysiol. 36, 179-190.

Polich, J. (1993). Cognitive brain potentials. Curr. Dir. Psychol. Sci. 2 175-262.

Ponton, C. W., Eggermont, J. J., Khosla, D., Kwong, B., and Don, M. (2002). Maturation of human central auditory system activity: separating auditory evoked potentials by dipole source modeling. Clin. Neurophysiol. 113 407-420.

Ponton, C. W., Eggermont, J. J., Kwong, B., and Don, M. (2000). Maturation of human central auditory system activity: evidence from multi-channel evoked potentials. Clin. Neurophysiol. $111,220-236$

Rogers, S. J., and Ozonoff, S. (2005). Annotation: what do we know about sensory dysfunction in autism? A critical review of the empirical evidence. J. Child Psychol. Psychiatry 46, 1255-1268.

Segalowitz, S. J. (1999). ERPScore Program: Peak and Area Analysis of Event-Related Potentials. St. Catharines, ON: Brock University.

Segalowitz, S. J., and Davies, P. L. (2004) Charting the maturation of the frontal lobe: an electrophysiological strategy. Brain Cogn. 55, 116-133.

Yochman, A., Ornoy, A., and Parush, S. (2006). Co-occurrence of developmental delays among preschool children with attention-deficit-hyperactivity disorder. Dev. Med. Child Neurol. 48, 483-488.

Conflict of Interest Statement: The authors declare that the research was conducted in the absence of any commercial or financial relationships that could be construed as a potential conflict of interest.

Received:01 April 2009; paperpending published: 11 August 2009; accepted: 04 May 2010; published online: 28 May 2010. Citation: Davies PL, Chang W-P and Gavin WJ (2010) Middle and late latency ERP components discriminate between adults, typical children, and children with sensory processing disorders. Front. Integr. Neurosci. 4:16. doi: 10.3389/fnint.2010.00016 Copyright (c) 2010 Davies, Chang and Gavin. This is an open-access article subject to an exclusive license agreement between the authors and the Frontiers Research Foundation, which permits unrestricted use, distribution, and reproduction in any medium, provided the original authors and source are credited. 